\title{
Diversity and conservation status of Peruvian palms
}

\author{
FRANCIS KAHN* and FARANA MOUSSA \\ ORSTOM, CP-09747, 70001-970 Brasília (DF), Brazil
}

Received 24 May 1993; revised and accepted 3 September 1993

Indigenous palm species of Peru are listed with data on their distribution patterns, ecology, frequency, density in the ecosystems, and conservation status. Peruvian palm flora includes 140 native species in 34 genera with the following distribution patterns: strictly Andean (17), Andean and Subandean (3), strictly Subandean (19), Subandean and Amazonian (20), strictly Amazonian (78), Amazonian and South peripheral (2), South peripheral (1). About $43 \%$ of the species occur at very low or low frequency in the country and about $9 \%$ are insufficiently known in situ for their conservation status to be defined. There are no Extinct species. Sixteen of the 17 strictly Andean palms are threatened species; 3 of them are Endangered, while only 5 strictly Subandean, 3 Subandean-Amazonian, and 4 strictly Amazonian palms are in these categories.

Keywords: Palmae; Peru; distribution patterns; ecology; conservation status

\section{Introduction}

Macbride (1960) published the only palm flora for Peru. It is now out of date. Shortly after its appearance, Harold Moore organized an expedition throughout Andean and Amazonian Peru (Moore et al., 1960). Since then, several genera have been revised: Geonoma (Wessels Boer, 1968), Chelyocarpus (Moore, 1972), Jessenia and Oenocarpus (Balick, 1986; Bernal et al., 1991), Hyospathe (Skov and Balslev, 1989), Dictyocaryum, Iriartea, Iriartella, and Socratea (Henderson, 1990), Ammandra and Phytelephas (Barfod, 1991), Astrocaryum (Kahn and Millán, 1992), Chamaedorea (Hodel, 1992), Aiphanes (Borchsenius and Bernal, in press); and two new genera were described, Itaya (Moore, 1972) and Aphandra (Barfod, 1991). Several taxonomic up-datings (Gentry, 1986; Kahn, 1990) and floristic inventories of palms in forest ecosystems (Kahn and Mejía, 1990, 1991; Kahn and Granville, 1992; Young 1992) were also published. In addition, countless specimens have been collected by R.B. Foster, A. Gentry, N. Jaramillo, F. Kahn, K. Mejía, D.N. Smith, R. Vásquez, K.R. Young, and many other botanists in the last fifteen years.

An analysis of a database including about 3000 herbarium specimens has provided information on species identification rates in each genus, species distribution patterns, and palm collecting intensity throughout Peru (Kahn et al., 1992). All specimens in each Subandean and Amazonian species have been listed according to the main river valley where they were collected, and never or poorly-collected areas were identified as a result (Moussa et al., 1992). 
A list of indigenous palm species of Peru is presented with data on their distribution patterns. ecology, trequency and density in the ecosystems. Their conservation status is defined from these parameters.

\section{Materials and methods}

\section{List of Peruvian palm species}

The list of indigenous palm species of Peru has been established from identification of herbarium specimens (AMAZ, BH. CUZ, K, MO, MOL, NY, P. US. USM - acronyms according to Holmgren et al., 1990, Index herbarorium. 8th ed.). It follows the checklist of Peruvian palms prepared by L. Brako, A. Henderson and F. Kahn, and revised by J. Dransfield, in Brako and Zarucchi's catalogue of flowering plants and gymnosperms of Peru (in press). Herbariurn voucher references will be found in this catalogue and in Kahn and Moussa (1.994).

\section{Distribution patterns of Perulian palms}

Five distribution patterns are proposed: (1) Strictly Andean species occur at high elevation $(>1501 \mathrm{~m})$ beyond the western limit of the Amazonian drainage which is marked by the occurrence of Dictyocaryum lamarckianum; Ceroxylon spp. are typically Andean palms, they reach the western piedmont of the Andes in northern Peru near the frontier with Ecuador: (2) Andean and Subandean species; (3) strictly Subandean species occupy the eastern piedmont of the Andes; (4) Amazonian species are found in the plain (strictly Amazonian). some of them reach the piedmont valleys below $1000 \mathrm{~m}$ (Subandean-Amazonian) or extend beyond the southern limit of Amazonia (Amazonian-South peripheral); (5) South peripheral species are found in Madre de Dios; they dominate tree vegetation in savannas and gallery forests which extend to Beni. Bolivia, and south of Rondonia. Brazil.

\section{Peruvian ecosystems with palms}

Ten ecosystems are considered: (i) Terra firme forests on clay, usually well-drained soils with the highest palm diversity; (ii) dry white sands with low vegetation and very low palm diversity; (iii) waterlogged white sands with high palm diversity; (iv) seasonal swamp forests irregularly flooded by rainfall with high density and medium diversity of palms: (v) permanently flooded swamp forests usually composed of very dense populations of Mauritia flexuosa, but a rather low diversity in palms; (vi) periodically flooded whitewater forests on alluvial soils (called restinga-forests in Peru, várzea-forests in Brazil) with medium palm diversity; (vii) forests periodically flooded by blackwater with low palm diversity (called tahuampa-forests in Peru, igapó-forests in Brazil); (viii) savannas and (ix) gallery forests, both with low palm diversity; $(x)$ mountain cloud forests above $1500 \mathrm{~m}$. Some species occur in forests flooded by whitewater as well as in those flooded by blackwater; they are considered 'riparian' in the list. The ability of some forest palms to flourish in deforested areas is noted under 'open vegetation.'

Descriptions of these ecosystems in Peru are given by Marmillod (1982), Encarnación (1985). Kalliola et al. (1987), Lamotte (1990), and Lopez and Freitas (1990) for Amazonian lowlands, and by Weberbauer (1945). Ferreyra (1950). ONERN (1976). Young and León (1988), and Young (1990) for eastern piedmont and the Andes. 
The ecosystem(s) given in the list is (are) that (those) where the species is commonly found. The attribution of only one ecosystem to a species does not exclude a certain ecological range within this species, which cannot be taken into account here. For instance, Astrocaryum javarense or Chelyocarpus repens form very dense stands on welldrained soils in terra firme forests; some individuals, however, may occur in the contiguous seasonal swamp forest.

More information on species richness, density, vertical distribution, life forms, and ecology of palms in Subandean and Amazonian forest ecosystems are proposed by Kahn and Granville (1992). Data on Andean palm flora will be found in Young (1992).

\section{Species ecology, frequency in Peru and density in ecosystems}

The attribution of the above-defined ecosystem(s) to each species, as well as the frequency and density evaluation are mainly based on the field experience of the senior author who has been working with palms for 8 years in Peru. The species which have not been seen in the field are mainly those which are known only from the type or from a few old specimens (most often destroyed at B). As far as possible, complementary information was supplied from specimen labels, ecological and floristic studies (Gentry, 1985; Young, 1992), and forest inventories. Many of the latter, however, do not take palms into account, and when they do, the frequent absence of herbarium references make most identifications questionable.

\section{Conservation status}

The following IUCN conservation categories (Dransfield et al., 1988) are employed: Extinct, Endangered, Vulnerable, Rare, Indeterminate for threatened categories; Status unknown, Insufficiently known for unknown categories; and non-threatened categories.

The conservation status of each species incorporates its distribution pattern because of the difference of human impact between regions, its frequency in the region, and its density in the ecosystem.

As a general rule species which occur in very low frequency and are not known from other countries, or are known in very low frequency therein, are included in threatened categories: (i) as Endangered when there is evidence that current populations are mere remnants of former larger populations under strong human pressure; (ii) as Vulnerable when population density is low and the deforestation rate is still high; (iii) as Rare when population density is high because the species generally grow in open areas and can persist under strong human pressure; (iv) as Indeterminate when human pressure is low.

Andean and Subandean species with low frequency and low density are also included in the threatened categories while Amazonian species with the same parameters are not. As a matter of fact, human pressure is very high in Andean and Subandean parts. Terrorism in the highlands made people migrate to the eastern Andes; mountain cloud forests have been intensively cut as a result. Many people were also attracted by coca cultivation on the piedmont where many areas have been deforested. Amazonian species, even with low frequency, may be considered not threatened as yet because of the lack of access roads and the consequently low density of human settlements in Amazonian lowlands.

The unknown categories include species which have long been described and, since then, have not been found again, e.g., Catoblastus pubescens, Geonoma congestissima, G. dicranospadix or Wettinia weberbaueri. 


\section{Results}

\section{Diversity of Peruvian palms}

A total of 140 native species in 34 genera has been listed (Table 1). Five species which are not indigenous palms of Peru are excluded: Bactris gasipaes. Elaeis guineensis, Euterpe oleracea, Oenocarpus bacaba, and Phoenix canariensis.

\section{Distribution patterns}

Strictly Amazonian palm species represent $55.7 \%$ of the 140 species listed; $13.6 \%$ of them are strictly Subandean species: $14.3 \%$ of them are common to Amazonian lowlands and Subandean peidmont. Andean species represent $12.1 \%$ of the total; only three species are common to Andean and Subandean regions. Two Amazonian species also occur beyond the southern limit of the basin (Amazonian-South peripheral palms); there is only one South peripheral species (Table 2).

\section{Ecosytems}

All Andean and several Subandean species occur in mountain cloud forests. A total of 70 Amazonian and Subandean species grow in terra firme forests, and 10 of them are also found in other ecosystems. There are 16 and 22 species in forests on periodically flooded alluvial soils and in seasonal swamp forests, respectively; six of the former and 12 of the latter present a wider ecological range, however. The other ecosystems have a few palm species, but these may be very abundant (Kahn and Granville, 1992). All species occurring in forests which are periodically flooded by blackwater, such as Astrocaryum jauari. Bactris maraja, B. riparia, are also found in most inundated forest types (see riparian species in Table 1). The ecology of five species is still unknown.

\section{Frequency and density}

Thirty two species occur at very low trequency. 29 at low frequency. On the whole, $43.6 \%$ of the palm species are scarce, and $70.5 \%$ of these are also found at low density. Frequency and density are unknown for 11 species.

\section{Distribution pattern and frequency}

All strictly Andean species occur at very low or low frequency (Table 2). Scarce species represent $90.0 \%, 45.2 \%$, and $31.0 \%$ of the palm flora of the Andes, the eastern piedmont, and Amazonian lowlands, respectively. The South peripheral species, Astrocaryum huaimi. was collected once in Peru, but it is abundant in the adjacent Bolivian region.

\section{Conservation status}

There are no Extinct palm species in Peru. Twenty eight $(20.0 \%)$ of the species are, however. listed as threatened; $9.3 \%$ are unknown: and $70.7 \%$ are not threatened.

Sixteen $(94.1 \%)$ strictly Andean species are within the threatened categories. Cero$x y$ lon latisectum, C. vermalosum, and $C$. weberbaueri are considered Endangered species because only small populations remain in sites where mountain forests are being intensively destroyed. Ceroxylon crispum, which is less scarce, is considered Vulnerable. Aiphanes spicata and Euterpe luminosa, two species described recently, and 10 species 


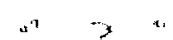

Table 1. Checklist of Peruvian palm species with their distribution patterns, ecology, frequency in the country, density in the ecosystem, and conservation status

\begin{tabular}{|c|c|c|c|c|c|}
\hline Species & $\begin{array}{l}\text { Distribution } \\
\text { pattern }^{\mathrm{a}}\end{array}$ & Ecosystem $^{\mathrm{b}}$ & $\begin{array}{l}\text { Frequency in } \\
\text { country }\end{array}$ & $\begin{array}{l}\text { Density in } \\
\text { ecosystem }^{c}\end{array}$ & $\begin{array}{l}\text { Conservation } \\
\text { status }^{\mathrm{d}}\end{array}$ \\
\hline Aiphanes aculeata Willdenow & SA/AM & $\mathrm{TF}$ & $\mathrm{L}$ & $\mathrm{L}$ & $\mathrm{nt}$ \\
\hline Aiphanes deltoidea Burret & $\mathrm{SA} / \mathrm{AM}$ & TF & $v L$ & $\mathrm{~L}$ & $\mathrm{R}$ \\
\hline $\begin{array}{l}\text { Aiphanes spicata } \\
\text { Borchsenius \& Bernal }\end{array}$ & A & $\mathrm{MF}$ & $v L$ & $\mathrm{~L}$ & $V^{e}$ \\
\hline Aiphanes ulei (Dammer) Burret & SA/AM & $\mathrm{TF}$ & $\mathrm{vL}$ & $\mathbf{L}$ & $\mathbf{R}$ \\
\hline Aiphanes weberbaueri Burret & SA/AM & TF/DWS & $\mathbf{L}$ & $\mathrm{L}$ & $\mathrm{nt}$ \\
\hline $\begin{array}{l}\text { Aphandra natalia } \\
\text { (Balslev \& Henderson) } \\
\text { Barfod }\end{array}$ & $\mathrm{AM}$ & $\mathrm{TF} / \mathrm{O}$ & $\mathrm{vL}$ & $\mathrm{H}$ & $n \mathrm{t}^{\mathrm{f}}$ \\
\hline $\begin{array}{l}\text { Astrocaryum carnosum } \\
\text { Kahn \& Millán }\end{array}$ & SA & PFAS & $v L$ & $\mathrm{H}$ & $\mathrm{R}^{\mathrm{e}}$ \\
\hline Astrocaryum chambira Burret & $\mathrm{AM}$ & TF/PFAS/O & $\mathrm{H}$ & $\mathrm{H}$ & nt \\
\hline Astrocaryum chonta Martius & $\mathrm{AM} / \mathrm{SP}$ & PFAS & $\mathbf{M}$ & $\mathrm{H}$ & nt \\
\hline $\begin{array}{l}\text { Astrocaryum gratum } \\
\text { Kahn \& Millán }\end{array}$ & $\mathrm{AM} / \mathrm{SP}$ & PFAS/GF & M & $\mathrm{H}$ & nt \\
\hline Astrocaryum huaimi Martius & $\mathrm{SP}$ & SAV & $\mathrm{vL}$ & $\mathrm{M}$ & $\mathrm{nt}^{\mathrm{f}}$ \\
\hline $\begin{array}{l}\text { Astrocaryum huicungo } \\
\text { Dammerex Burret }\end{array}$ & SA & $\mathrm{SSF} / \mathrm{O}$ & $\mathrm{vL}$ & $\mathrm{H}$ & $\mathrm{R}^{\mathrm{e}}$ \\
\hline Astrocaryum jauari Martius & $\mathrm{AM}$ & $\mathbf{R}$ & $\mathrm{H}$ & $\mathrm{M} / \mathrm{H}$ & $\mathrm{nt}$ \\
\hline $\begin{array}{l}\text { Astrocaryum javarense } \\
\text { Trail ex Drude }\end{array}$ & $\mathrm{AM}$ & $\mathrm{TF}$ & $\mathbf{M}$ & $\mathrm{H}$ & $\mathrm{nt}$ \\
\hline $\begin{array}{l}\text { Astrocaryum macrocalyx } \\
\text { Burret }\end{array}$ & $\mathrm{AM}$ & $\mathrm{TF}$ & $\mathrm{L}$ & $\mathrm{H}$ & $\mathrm{nt}$ \\
\hline $\begin{array}{l}\text { Astrocaryum perangustatum } \\
\text { Kahn \& Millán }\end{array}$ & SA & $\mathrm{TF} / \mathrm{O}$ & $\mathrm{vL}$ & $\mathrm{H}$ & $\mathrm{R}^{\mathrm{e}}$ \\
\hline $\begin{array}{l}\text { Astrocaryum scopatum } \\
\text { Kahn \& Millán }\end{array}$ & $\mathrm{AM}$ & PFAS & $\mathrm{vL}$ & $\mathrm{H}$ & $\mathrm{R}^{\mathrm{e}}$ \\
\hline Attalea tessmannii Burret & $\mathrm{AM}$ & $\mathrm{TF}$ & $?$ & $?$ & $?$ \\
\hline $\begin{array}{l}\text { Bactris acanthocarpoides } \\
\text { Barbosa Rodrigues }\end{array}$ & $\mathrm{AM}$ & $\mathrm{TF}$ & $\mathbf{M}$ & $\mathrm{L} / \mathrm{M}$ & $\mathrm{nt}$ \\
\hline
\end{tabular}




\begin{tabular}{|c|c|c|c|c|c|}
\hline Species & $\begin{array}{l}\text { Distribution } \\
\text { pattern }^{\text {a }}\end{array}$ & Ecosystend & $\begin{array}{l}\text { Frequency in } \\
\text { country }^{2}\end{array}$ & $\begin{array}{l}\text { Density in } \\
\text { ecosystem }\end{array}$ & $\begin{array}{l}\text { Conservation } \\
\text { status }^{\mathrm{d}}\end{array}$ \\
\hline $\begin{array}{l}\text { Bactris acanthospatha } \\
\text { Trail ex Drude }\end{array}$ & $\mathrm{AM}$ & $\mathrm{TF}$ & $M$ & M & $\mathrm{nt}$ \\
\hline Bactris angustifolia Dammer & $\mathrm{AM}$ & $?$ & $?$ & ? & $?$ \\
\hline $\begin{array}{l}\text { Buctris anundinacea } \\
\text { (Trail) Drude }\end{array}$ & $\mathrm{AM}$ & $\mathbf{R}$ & $\mathrm{M}$ & M & $\mathrm{nt}$ \\
\hline Bactris bifida Martius & AM & PFAS & M & $\mathrm{H}$ & $\mathrm{nt}$ \\
\hline $\begin{array}{l}\text { Bactris chlorancatha } \\
\text { Poeppig ex Martius }\end{array}$ & $\mathrm{AM}$ & $?$ & $?$ & $?$ & $?$ \\
\hline Bactris concinna Martius & $\mathrm{AM}$ & $\mathrm{R}$ & M & $\mathrm{M}$ & $\mathrm{nt}$ \\
\hline Bactris corossilla Karsten & $\mathrm{AM}$ & SSF & $\mathrm{vL}$ & $\mathrm{L}$ & $n t^{f}$ \\
\hline Bactris fissifrons Martius & $\mathrm{AM}$ & TF & $?$ & $?$ & $?$ \\
\hline Bactris hirta Martius & Am & $\mathrm{TF}$ & $\mathrm{M}$ & $\mathrm{L}$ & $\mathrm{nt}$ \\
\hline $\begin{array}{l}\text { Bactris humilis } \\
\text { (Wallace) Burret }\end{array}$ & AM & $\mathrm{TF}$ & $\mathrm{M}$ & $\mathrm{M}-\mathrm{H}$ & $\mathrm{nt}$ \\
\hline Bactris killipii Burret & $\mathrm{AM}$ & $\mathrm{TF}$ & $L$ & $L$ & $\mathrm{nt}$ \\
\hline Bactris maraja Martius & $\mathrm{AM}$ & $\mathrm{R}$ & $\mathrm{H}$ & M & $\mathrm{nt}$ \\
\hline $\begin{array}{l}\text { Bactris monticola } \\
\text { Barbosa Rodrigues }\end{array}$ & $\mathrm{AM}$ & SSF & $\mathrm{H}$ & $\mathrm{H}$ & nt \\
\hline Bactris piranga Trail & $\mathrm{AM}$ & $\mathrm{TF}$ & $\mathrm{L}$ & $\mathrm{L}$ & $\mathrm{nt}$ \\
\hline Bactris riparia Martius & $\mathrm{AM}$ & $\mathrm{R}$ & M & M & nt \\
\hline $\begin{array}{l}\text { Bactris simplicifrons } \\
\text { Martius }\end{array}$ & SA/AM & $\mathrm{TF}$ & $\mathrm{H}$ & $\mathrm{L}$ & nt \\
\hline Bactris sphaerocarpa Trail & AM & $\mathrm{TF}$ & $\mathrm{M}$ & $\mathrm{H}$ & $\mathrm{nt}$ \\
\hline $\begin{array}{l}\text { Bactris utilis (Oersted) } \\
\text { Bentham \& Hooker f. } \\
\text { ex Hemsley }\end{array}$ & $\mathrm{SA}$ & $\mathrm{O}$ & M & $\mathrm{L}$ & $\mathrm{nt}$ \\
\hline $\begin{array}{l}\text { Catoblastus drudei } \\
\text { Cook \& Doyle }\end{array}$ & $\mathrm{AM}$ & $\mathrm{TF}$ & $\mathrm{L}$ & $M$ & $n t^{t}$ \\
\hline $\begin{array}{l}\text { Catoblastus pubescens } \\
\text { (Karsten) Wendland }\end{array}$ & $\mathrm{AM}$ ? & $?$ & $?$ & $?$ & $?$ \\
\hline Ceroxylon crispum Burret & A & MF & $\mathrm{L}$ & $\mathbf{L}$ & V \\
\hline Ceroxylon latisectum Burret & A & MF & $\mathrm{vL}$ & $\mathrm{L}$ & $E^{e}$ \\
\hline
\end{tabular}


Ceroxylon verruculosum

\section{Burret}

Ceroxylon weberbaueri Burret

Chamaedorea angustisecta

Burret

Chamaedorea fragrans

(Ruiz \& Pavón) Martius

Chamaedorea linearis

(Ruiz \& Pavón) Martius

Chamaedorea pauciflora

Martius

Chamaedorea pinnatifrons

(Jacquin) Oersted

Chelyocarpus repens

Kahn \& Mejía

Chelyocarpus ulei Dammer

Desmoncus leptospadix

Martius

Desmoncus longifolius

Martius

Desmoncus mitis

Martius

Desmoncus orthacanthos

Martius

Desmoncus polyacanthos

Martius

Desmoncus setosus Martius

Desmoncus vacivus Bailey

Dictyocaryum lamarckianum

(Martius) Wendland

Dictyocaryum ptariense

(Steyermark)

Moore \& Steyermark Elaeis oleifera

(Kunth) Cortés

Euterpe catinga Wallace

\section{A}

A

SA/AM

$\mathrm{vL}$

L

$E^{e}$

SA

TF

$\mathrm{vL}$

L

$E^{e}$

L

nt

SA

$\mathrm{TF}$

L

L

nt

SA

TF

L

L

nt

SA/AM

TF

M

$\mathrm{H}$

nt

SA/AM $\quad$ SSF

TF

AM

SSF

SA/AM

AM

PFAS

AM

$\mathrm{O}$

AM

$\mathrm{R} / \mathrm{O}$

TF

L.

H

nt

$v L$

H

$\mathrm{I}^{\mathrm{e}}$

vL

H

L

I

$\mathrm{M}$

L

L

nt

K

AM

AM

AM

AM

A

AM

AM

AM
SSF

WWS

PFAS/O/R H

TF

O/R

MF
L

L

$v \mathrm{~L}$

M

R

nt

nt

nt

nt

nt

nt

$\mathrm{nt}$

nt

M

H

H 


\begin{tabular}{|c|c|c|c|c|c|}
\hline Species & $\begin{array}{l}\text { Distribution } \\
\text { pattern }^{\text {a }}\end{array}$ & Ecosystem $^{b}$ & $\begin{array}{l}\text { Frequency in } \\
\text { country }\end{array}$ & $\begin{array}{l}\text { Density in } \\
\text { ecosystem }\end{array}$ & $\begin{array}{l}\text { Conservation } \\
\text { status }^{\text {d }}\end{array}$ \\
\hline $\begin{array}{l}\text { Euterpe luminosa } \\
\text { Henderson, Galeano } \\
\text { \& Mesa }\end{array}$ & $A$ & $\mathrm{MF}$ & $v \mathbf{L}$ & $\mathrm{L}$ & V \\
\hline Euterpe precatoria Martius & AM & SSF & $\mathrm{H}$ & $\mathrm{H}$ & nt \\
\hline Geonoma acaulis Martius & AM & $\mathrm{SSF} / \mathrm{PSF}$ & $\mathrm{H}$ & $\mathrm{H}$ & nt \\
\hline $\begin{array}{l}\text { Geonoma andicola } \\
\text { Dammer ex Burret }\end{array}$ & $\mathrm{A}$ & MF & $v L$ & $\mathbf{L}$ & $V^{e}$ \\
\hline Geonoma arundinacea Martius & AM & TF & $\mathrm{M}$ & $\mathrm{M}$ & nt \\
\hline Geonoma aspidiifolia Spruce & AM & WSS & $\mathrm{L}$ & $\mathrm{L}$ & $\mathrm{K}$ \\
\hline $\begin{array}{l}\text { Geonoma baculifera } \\
\text { (Poiteau) Kunth }\end{array}$ & $\mathrm{AM}$ & $\mathrm{SSF}$ & $?$ & $?$ & $11 t^{\mathrm{g}}$ \\
\hline Geonoma bartlettii Burret & AM & TF & $v \mathbf{L}$ & $\mathrm{L}$ & $\mathrm{k}$ \\
\hline Geonoma bartlettii Dammerex Burret & $\mathrm{SA}$ & SSF & $\mathrm{M}$ & $\mathrm{H}$ & nt \\
\hline Geonoma brongniartii Martius & AM & $\mathrm{SSF} / \mathrm{TF}$ & $\mathbf{M}$ & M & nt \\
\hline Geonoma camana Trail & $\mathrm{SA}$ & $\mathrm{TF}$ & $?$ & $?$ & $?$ \\
\hline $\begin{array}{l}\text { Geonoma congestissina Burret } \\
\text { Geonoma cuneata }\end{array}$ & AM & TF & $\mathrm{L}$ & $\mathrm{L}$ & nt \\
\hline Wendland ex Spruce & AM & $\mathrm{TF}$ & $\mathbf{M}$ & $\mathrm{L}$ & $\mathrm{nt}$ \\
\hline Geonoma decurrens & & & & & \\
\hline $\begin{array}{l}\text { Wendland et Burret } \\
\text { Linden ex Wendland }\end{array}$ & A & $\mathrm{MF}$ & $\mathrm{VL}$ & $\mathrm{L}$ & $\mathrm{V}$ \\
\hline $\begin{array}{l}\text { Geonoma deversa } \\
\text { (Poiteau) Kunth }\end{array}$ & AM & TF & $\mathrm{H}$ & M & $\mathrm{nt}$ \\
\hline $\begin{array}{l}\text { Geonoma dicranospadix } \\
\text { Burret }\end{array}$ & SA & $?$ & $?$ & $?$ & $?$ \\
\hline $\begin{array}{l}\text { Geonoma ferruginea } \\
\text { Wendland ex Spruce }\end{array}$ & $\mathrm{SA}$ & TF & $\mathrm{L}$ & $\mathrm{L}$ & $\mathrm{nt}$ \\
\hline $\begin{array}{l}\text { Geonoma gracipiles } \\
\text { Dammer ex Burret }\end{array}$ & $\mathrm{SA}$ & $\mathrm{TF}$ & $?$ & $?$ & $?$ \\
\hline Geonoma granditrijuga Burret & $\mathrm{A} / \mathrm{SA}$ & $\mathrm{MF}$ & $\mathbf{M}$ & $\mathrm{M}$ & nt \\
\hline Geonoma helminthoclada & A & $\mathrm{MF}$ & $V L$ & $\mathrm{~L}$ & V \\
\hline
\end{tabular}


Geonoma juruana Dammer

Geonoma jussieuana Martius

Geonoma laxiflora Martius

Geonoma lehmannii

Dammer ex Burret

Geonoma leptospadix Trail

Geonoma lindeniana Wendland

Geonoma macrostachys Martius

Geonoma marggraffia Engel

Geonoma maxima

(Poiteau) Kunth

Geonoma megalospatha Burret

Geonoma oligoclona Trail

Geonoma piscicauda Dammer

Geonoma poeppigiana Martius

Geonoma pycnostachys Martius

Geonoma spixiana Martius

Geonoma tamandua Trail

Geonoma tessmannii Burret

Geonoma trailii Burret

Geonoma triglochin Burret

Geonoma trigona

(Ruiz \& Pavón) Gentry

Geonoma undata Klotzsch

Geonoma weberbaueri

Dammer ex Burret

Hyospathe elegans Martius

Hyospathe ulei Dammer

Iriartea deltoidea

Ruiz \& Pavón

Iriartella stenocarpa Burret

Itaya amicorum Moore

Lepidocaryum gracile Martius
AM

SA

AM

A

AM

A

AM

A

AM

A

AM

AM

AM

AM

AM

AM

AM

AM

SA

A

A

A

AM

SA

SA/AM

TF

MF/SSF

PFAS

MF

TF

MF

SSF/PFAS

MF

TF

MF

TF/SSF

TF

TF

TF

TF

SSF

TF

DWS

TF

MF

MF

MF

TF/SSF

TF

TF/MF

TF

AM

AM

PFAS

TF

AM
M

nt

nt

nt

$\mathrm{nt}$

$\mathrm{M}$

$\mathrm{vL}$

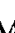

L

M

L

M

$\mathrm{nt}$

$\mathrm{V}$

nt

nt

V

nt

nt

$\mathrm{nt}$

nt

nt

nt

K

nt

nt

$\mathrm{V}^{\mathrm{e}}$

V

vL

vL

L

L

$\mathrm{H}$

L

$\mathrm{H}$

nt

vL

M

$\mathrm{H}$

$\mathrm{L}$
$\mathrm{M}$

$\mathrm{H}$
$\mathrm{H}$ 


\begin{tabular}{|c|c|c|c|c|c|c|}
\hline Species & $\begin{array}{l}\text { Distribution } \\
\text { pattern }^{\mathrm{a}}\end{array}$ & Ecosystem ${ }^{\mathrm{b}}$ & $\begin{array}{l}\text { Frequency in } \\
\text { country }\end{array}$ & $\begin{array}{l}\text { Density in } \\
\text { ecosystem }\end{array}$ & $\begin{array}{l}\text { Conservation } \\
\text { status }^{\mathrm{d}}\end{array}$ & $\partial$ \\
\hline Lepidocaryum tenute Martius & AM & $\mathrm{TF}$ & $M$ & M & nt & \\
\hline $\begin{array}{l}\text { Manicaria saccifera } \\
\text { Gaertner }\end{array}$ & $\mathrm{AM}$ & SSF & $\mathrm{L}$ & M & $\mathrm{nt}$ & \\
\hline Mauritia carana Wallace & AM & WWS & $\mathrm{L}$ & $\mathrm{H}$ & $\mathrm{nt}$ & \\
\hline Mauritia flexuosa L.f. & SA/AM & SSF $/ \mathrm{PSF}$ & $\mathrm{rH}$ & $\mathrm{vH}$ & $n t$ & \\
\hline $\begin{array}{l}\text { Mauritiella aculeata } \\
\text { (Kunth) Burret }\end{array}$ & $\mathrm{AM}$ & DWS & $\mathbf{M}$ & $\mathrm{M}$ & $\mathrm{nt}$ & \\
\hline $\begin{array}{l}\text { Maximiliana maripa } \\
\text { (Aublet) Drude }\end{array}$ & $\mathrm{AM}$ & TF & $\mathrm{M}$ & $\mathrm{M}-\mathrm{H}$ & $\mathrm{nt}$ & \\
\hline Oenocarpus balickii Kahn & $\mathrm{AM}$ & TF & $\mathrm{M}$ & M & $n t$ & \\
\hline Oenocarpus bataua Martius & $\mathrm{SA} / \mathrm{AM}$ & SSF/WWS/O & $\mathrm{H}$ & $\mathrm{H}$ & nt & \\
\hline Oenocarpus mapora Karsten & $\mathrm{SA} / \mathrm{AM}$ & SSF/PSF/PFAS & $\mathrm{H}$ & $\mathrm{H}$ & $\mathrm{nt}$ & \\
\hline Oenocarpus minor Martius & $\mathrm{AM}$ & TF & $V L$ & $\mathrm{~L}$ & $n t^{\mathrm{g}}$ & \\
\hline Orbignya polysticha Burret & $\mathrm{AM}$ & TF & $\mathrm{H}$ & $\mathrm{H}$ & $\mathrm{nt}^{\mathrm{b}}$ & \\
\hline $\begin{array}{l}\text { Orbignya racemosa } \\
\text { (Spruce) Drude }\end{array}$ & $\mathrm{AM}$ & $\mathrm{TF}$ & $\mathrm{L}$ & $\mathrm{L}$ & $\mathrm{nt}^{\mathrm{h}}$ & \\
\hline $\begin{array}{l}\text { Pholidostachys synanthera } \\
\text { (Martius) Moore }\end{array}$ & $\mathrm{SA} / \mathrm{AM}$ & $\mathrm{TF}$ & $\mathrm{H}$ & $\mathrm{H}$ & nt & \\
\hline $\begin{array}{l}\text { Phytelephas macrocarpa } \\
\text { Ruiz \& Pavón }\end{array}$ & $\mathrm{SA} / \mathrm{AMI}$ & PFASSSF & $\mathrm{M}$ & $\mathrm{H}$ & $\mathrm{nt}$ & \\
\hline $\begin{array}{l}\text { Prestoea actuminata } \\
\text { (Willdenow) Moore }\end{array}$ & $\mathrm{A} / \mathrm{SA}$ & $\mathrm{MF}$ & $\mathrm{M}$ & $\mathrm{L}$ & $\mathrm{nt}$ & \\
\hline $\begin{array}{l}\text { Prestoea ensiformis } \\
\text { (Ruiz \& Pavón) Moore }\end{array}$ & $\mathrm{A} / \mathrm{SA}$ & $\mathrm{MF}$ & L & $\mathrm{L}$ & $\mathrm{nt}$ & \\
\hline $\begin{array}{l}\text { Prestoea schultzeana } \\
\text { (Burret) Moore }\end{array}$ & $S A$ & $\mathrm{TF}$ & $\mathrm{L}$ & $\mathrm{L}$ & $\mathrm{nt}$ & $\hat{\Xi}$ \\
\hline $\begin{array}{l}\text { Scheelea butyracea } \\
\text { (Mutis ex L.f.) Karsten } \\
\text { ex Wendland }\end{array}$ & $\mathrm{AM}$ & PFAS & $\mathrm{M}$ & M & $\mathrm{nt}$ & $\underset{3}{3}$ \\
\hline $\begin{array}{l}\text { Scheelea insignis } \\
\text { (Martius) Karsten }\end{array}$ & $\mathrm{AM}$ & $\mathrm{TF}$ & $\mathrm{M}$ & $\mathrm{H}$ & $\mathrm{nt}$ & $\stackrel{\Xi}{a}$ \\
\hline
\end{tabular}




\section{Burret}

Socratea exorrhiza

(Martius) Wendland Socratea salazarii Moore

Syagrus sancona Karsten Syagrus smithii

(Moore) Glassman

Welfia georgii

Wendland ex Burret

Wendlandiella gracilis

\section{Dammer}

Wettinia augusta

Poeppig \& Endlicher

Wettinia longipetala Gentry

Wettinia maynensis Spruce

Wettinia weberbaueri Burret
$\mathrm{AM}$

TF/S-PSF/PFAS vH

$\mathrm{M} / \mathrm{H}$

nt

SA/AM TF

SA/AM TF

AM

$\mathrm{TF}$

SA

MF

AM

$\mathrm{TF}$

SA/AM

$\mathrm{TF}$

$\mathrm{H}$

M

L

M

M

L

$n t^{e}$

nt

nt

SA

SA

TF

SA
vL

L

M

L

H

M

vL

M

?

${ }^{a}$ Distribution patterns - A: Strictly Andean species; A/SA: Andean-Subandean species; AM: Strictly Amazonian species; AM/SP: Amazonian-South peripheral species; SA: Strictly Subandean species; SA/AM: Subandean-Amazonian species; SP: South peripheral species.

'Ecosystems - DWS: Dry white sands; GF: Gallery forests; MF: Mountain cloud forests; O: Open vegetation; PFAS: Forests on periodically flooded alluvial soils (várzea-forest); PSF: Permanent swamp forests (Mauritia flexuosa swamps); R: Riparian species (including forests periodically flooded by blackwater); SAV: Savannas; SSF: Seasonal swamp forests; TF: Terra firme forests; WWS: Waterlogged white sands; ?: Parameter unknown.

cFrequency and density - vL: very low; L: low; M: medium; H: high; vH: very high; ?: parameter unknown.

${ }^{\mathrm{d}}$ Conservation status - E: Endangered; V: Vulnerable; R: Rare; I: Indeterminate; ?: Status unknown; K: Insufficiently known; nt: Not threatened.

eEndemic species.

${ }^{\mathrm{f}}$ These species, rarely collected in Peru, are not included in the threatened categories because they occur at higher frequencies in other countries. Aphandra natalia has been reported by Mejía (1992) for Peru; it has also been collected near the Peruvian frontier in Ecuador (Borgtoft Pedersen, 1992), as well as in Acre, Brazil (Henderson 1126, NY). Astrocaryum huaimi has been collected in Madre de Dios, Peru (Albán 6936, USM). Bactris utilis is considered a synonym of $B$. gasipaes (Glassman, 1972).

SThese species are not likely to occur in Peru - Geonoma baculifera and Oenocarpus minor are common palms in eastern and central Amazonia, respectively.

${ }^{\mathrm{h}}$ These species are treated under genus Attalea in Brako and Zarucchi (in press). 
Table 2. Diversity of Peruvian palms, number of species with very low and low frequency, and number of species in IUCN categories in relation to distribution patterns. (Legend: see Table 1)

\begin{tabular}{|c|c|c|c|c|c|c|c|c|c|c|}
\hline \multirow{3}{*}{$\begin{array}{l}\text { Distribution } \\
\text { patterns }\end{array}$} & \multirow{3}{*}{$\begin{array}{l}\text { Number } \\
\text { of } \\
\text { Species }\end{array}$} & & & \multicolumn{7}{|c|}{ Conservation status } \\
\hline & & \multicolumn{2}{|c|}{ Frequency } & \multicolumn{3}{|c|}{$\begin{array}{l}\text { Threatened } \\
\text { categories }\end{array}$} & & \multirow{2}{*}{$\begin{array}{l}\text { Not } \\
\text { threatened } \\
\text { nt }\end{array}$} & \multicolumn{2}{|c|}{ Unknown } \\
\hline & & $v L$ & $\mathrm{~L}$ & $E$ & $V$ & $\mathrm{R}$ & I & & $?$ & $K$ \\
\hline Strictly Andean & 17 & 15 & 2 & 3 & 13 & 0 & 0 & 1 & 0 & 0 \\
\hline Andean/Subandean & 3 & 0 & 1 & 0 & 0 & 0 & 0 & 3 & 0 & 0 \\
\hline Strictly Subandean & 19 & 6 & 5 & 0 & 2 & 3 & 0 & 10 & 4 & 0 \\
\hline $\mathrm{SA} / \mathrm{AM}$ & 20 & 3 & 4 & 0 & (1) & 2 & 1 & 17 & 0 & 0 \\
\hline Amazonian AM & 78 & 7 & 17 & 0 & 0 & 2 & 2 & 65 & 5 & 4 \\
\hline AM/SP & 2 & 0 & 0 & 0 & 0 & 0 & 0 & 2 & 0 & 0 \\
\hline South peripheral & 1 & 1 & 0 & 0 & 0 & 0 & 0 & 1 & 0 & 0 \\
\hline Total & 140 & 32 & 29 & 3 & 15 & 7 & 3 & 99 & 9 & 4 \\
\hline
\end{tabular}

of Geonoma which grow in mountain cloud forests at high elevation are also Vulnerable. Most of these last occur from Venezuela to Bolivia (Wessels Boer, 1968; Glassman, 1972: Balslev and Moraes, 1989); the Vulnerable status may be severe. However, it combines (i) the high fragility of mountain forest ecosystems, (ii) the strong human pressure in these regions, and (iii) the fact that such understorey species are unable to survive in deforested areas. Geonoma lehmannii, $G$. lindeniana, and $G$. marggraffia have been classified as Vulnerable for Colombia (Bernal. 1989). Dictyocaryum lamarckianum is the only Andean species within the non-threatened categories.

The situation is not so serious for the strictly Subandean species. Only 5 of the 19 species are classified in the threatened categories: two of them. Hyospathe ulei and Wettinia longipetala, are Vulnerable. Four species are within the unknown categories. and ten are safe.

Only $7.1 \%$ of the species which occur in Amazonia are considered threatened. However. 9 species which are not sutficiently known yet to be classified are likely to increase those threatened categories.

\section{Endemism}

Endemic palms include 14 species (Table 1): six of them are strictly Andean species ( 3 Endangered and 3 Vulnerable): five are strictly Subandean species (1 Vulnerable, 3 Rare and 1 Unknown): one is a Subandean-Amazonian species (not threatened); and two are strictly Amazonian species (Indeterminate and Rare).

\section{Discussion}

\section{Peruvian palm diversity}

The high generic diversity of Peruvian palms is mainly due to the diversity of the Amazonian region. Thirty two of the 38 palm genera found in the Amazon basin and 
the Guianas are present in Peru (Kahn and Granville, 1992). Only Ceroxylon and Welfia are not found in Amazonia.

The diversity at the specific level is certainly high. However, such data are clearly dependent on the degree of confidence of species identification. This can be considered high for those species belonging to the above-mentioned genera which have been revised recently. It is not the case of many species of some genera which do need re-assessment, such as Bactris which presents a low rate $(55 \%)$ of identified vouchers (Kahn et al., 1992).

The preceding authors also pointed out that many species of Bactris (about $30 \%$, considering new taxonomic arrangements by A. Henderson, personal communication) and Geonoma (more than $50 \%$ ) are represented by only one or a few specimens. If several species are found to have been infrequently collected, it cannot be excluded that others have been erroneously named. The material is not complete enough to check the identification with confidence in many cases. This suggests that the species list proposed here is longer than it will be when the Peruvian palm flora is updated. On the other hand, several new species have been described (Gentry, 1986; Kahn and Mejía, 1988; Kahn, 1990; Henderson et al., 1991; Kahn and Millán, 1992; Borchsenius and Bernal, in press) and many species were reported new for Peru (Kahn and Mejía, 1986, 1990, 1991) in the last years. For instance, Astrocaryum huaimi, Mauritia carana and Manicaria saccifera were recently collected. Moreover, many locations in Peruvian Amazonia have not been explored yet, as already stated. On the whole, erroneous names will be replaced by those of the species newly described or reported for the country, and Peruvian palm diversity is not likely to decrease significantly as a result.

\section{Future of Peruvian palms}

Andean species are nearly all threatened. Current activities are for an increasing rate of deforestation. Chamaedorea and Geonoma are small palms in the understorey of mountain forests and will disappear with the forest. Ceroxylon are tall palms which persist in open areas, but they are commonly used as building material and consequently cut down. There are only a few roads crossing the Andes; the situation is still not catastrophic. When the economy of Peru recovers, new roads will be built to develop the country, this is an unescapable need for Peru, and that will be the end of many Andean species.

The situation will be similar in Amazonia. There are few threatened species because of the low density of human settlements. When roads and colonization extend through Amazonian lowlands, vast areas will be deforested and many species will become scarcer and scarcer. The striking fact is that $43.6 \%$ of the palm flora occur at low frequency (53\% including unknown species, which are likely to be still unknown because their frequency is very low). It is easy to imagine the example of Brazilian Rondônia being transposed to the Peruvian Amazonia resulting in a similar ecological catastrophe.

\section{Acknowledgements}

This work was supported by the international agreement between ORSTOM, France and Museum of Natural History (UNMSM), Lima, Peru, with grant WWF-US 3322. We are particularly indebted to John Dransfield, A. Henderson, Dennis V. Johnson, and 
Eduardo Lleras for their valuable criticisms of the manuscript. We also thank L. Brako, M. Hoff, K. Mejía. B. Millán who have contributed to the project.

\section{References}

Balick. M.J. (1986) Systematics and economic botany of the Oenocarpus-Jessenia (Palmae) complex. Adr. Econ. Bot. 3, 1-140.

Balslev. H. and Moraes. M. (1989) Sinopsis de las palmeras de Bolivia. Aarhus: AAU Reports 20.

Barfod, A.S. (1991) A monographic study of the subfamily Phytelephantoideae (Arecaceac). Opera Bot. 105, 1-73.

Bernal, R. (1989) Endangerment of Colombian palms. Principes 33. 113-28.

Bernal. R. Galeano, G. and Henderson, A. (1991) Notes on Oenocarpus in the Colombian Amazon. Brittonia 43, 154-64.

Borchsenius, F.K. and Bernal, R. (in press) A monograph of Aiphanes (Palmae). New York: Flora Neotropica Monograph.

Borgtoft Pedersen. H. (1992) Uses and management of Aphandra natalia (Palmae) in Ecuador. Bull. Inst. fr. eit. andines 21, 741-53.

Brako. L. and Zarucchi, J. (in press) Catalogue of the flowering plants and gymnosperms of Peru. Missouri Botanical Garden.

Dransfield, J.. Johnson. D. and Synge, H. (1988) The palms of the new world: A conservation census. Cambridge: IUCN-WWF Plants Conservation Programme, Publication No. 2.

Encarnación. F. (1985) Introducción a la flora y vegetación de la Amazonia peruana: estadio actual de los estudios, medio natural y ensayo de una clave de determinación de las formaciones vegetales en la llanura amazónica. Candollea 40, 237-52.

Ferreyra, R. (1950) Informe botánico de la explotación cientifica al valle del Huallaga. In Informe sobre el Huallaga pp. 177-216. Lima: OCHOA. Ministerio de Relaciones Exteriores.

Gentry, A.H. (1985) Algunos resultados preliminares de estudios botánicos en el Parque Nacional del Manu. In Report Manu pp. 1-23. Lima: Universidad Nacional Agraria.

Gentry. A.H. (1986) Notes on Peruvian palms. Anm. Missouri Bot. Gard. 73, 158-65.

Glassman. S.F. (1972) A revision of B. E. Dahlgren's index of American palms. Lehre: J Cramer.

Henderson. A. (1990) Arecaceae. Part I. Introduction and the Iriarteinat. New York: Flora Neotropica Monograph 53.

Henderson, A.. Galeano, G. and Mesa, E. (1991) A new species of Euterpe (Palmae) from Peru. Brittonia 43, 178-80.

Hodel, D.R. (1992) (hamaedorea. Lawrence, Kansas: International Palm Society, Allen Press.

Holmgren, P.K., Keuken. W. and Schofield, E.K. (1990) Index Herbarorium Part I. The herbaria of the world. Edn. 8. Utrecht: Bohn, Schettema and Holkema.

Kahn, F. (1990) Las palmeras del Arboretum Jenaro Herrera (Provincia de Requena, Departamento de Loreto. Perú). Contribución al estudio de la flora y de la vegetación de la Amazonia peruana. XVII. Candollea 45, 341-62.

Kahn, F, and Granville, J.J. de (1992) Palms in forest ecosystems of Amazonia. Berlin: Springer Verlag. Ecological Series, Volume 95.

Kahn. F. and Mejia, K. (1986) The American oil palm. Elaeis oleifera, in Peruvian Amazonia. Principes 30, 182.

Kahn. F. and Mejia, K. (1988) A new species of Chelyocarpus (Palmae Coryphoidede) from Peruvian Amazonia. Principes 32, 69-72.

Kahn, F. and Mejia, K. (1990) Palm communities in wetland forest ecosystems of Peruvian Amazonia. For. Ecol. Manag. 33/34, 169-79.

Kahn. F. and Mejia, K. (1991) The palm communities of two "terra firme" forests in Peruvian Amazonia. Principes 35, 22-6. 
Kahn, F. and Millán, B. (1992) Astrocaryum (Palmae) in Amazonia. A preliminary treatment. Bull. Inst. fr. ét. andines 21, 459-531.

Kahn, F. and Moussa, F. (1994) Las palmeras del Perú-colecciones, Patrones de distribución geográfica, Ecología, Estatutos de conservación, Nombres vernáculos, Utilizaciones. Lima: Travaux de l'Institut français d'études andines, Volume 59.

Kahn, F., Henderson, A., Brako, L., Hoff, M. and Moussa, F. (1992) Datos preliminares a la actualización de la flora de Palmae del Perú: intensidad de herborización y riqueza de las colecciones. Bull. Inst. fr. ét. andines 21, 549-63.

Kalliola, R., Salo, J. and Mäkinen, Y. (1987) Regeneración natural de selvas en la Amazonia peruana. 1. Dinámica fluvial y sucesión ribereña. Lima: UNMSM.

Lamotte. S. (1990) Fuvial dynamics and succession in the lower Ucayali basin, Peruvian Amazonia. For. Ecol. Manag. 33/34, 141-56.

López Parodi, J. and Freitas, D. (1990) Geographical aspects of forested wetlands in the lower Ucayali, Peruvian Amazonia. For. Ecol. Manag. 33/34, 157-68.

Macbride, J.F. (1960) Flora of Peru. Part I, No. 2, Palmae. Field. Mus. Nat. Hist. Bot. 13, 321418.

Marmillod, D. (1982) Methodik und Ergebnisse von Untersuchungen über Zusammensetzung und Aufbau eines Terrassenwaldes in peruanischen Amazonien. Göttingen: Thesis Georg-August Univ.

Mejía, K. (1992) Las palmeras en los mercados de Iquitos. Bull. Inst. fr. ét. andines 21, 755-69.

Moore, H.E. Jr., (1972) Chelyocarpus and its Allies Cryosophila and Itaya (Palmae). Principes 16, 67-88.

Moore, H.E. Jr., Salazar, A.C. and Smith, E.E. (1960) A reconnaissance survey of palms in eastern Peru. Lima: Agriculture division, United States operation mission to Peru, International cooperation administration (unpublished).

Moussa. F., Kahn, F., Henderson, A., Brako, L. and hoff, M. (1992) Las palmeras en los valles principales de la Amazonia peruana. Bull. Inst. fr. ét. andines 21, 565-97.

ONERN (1976) Mapa ecológico del Perú y guia explicativa. Lima: Oficina Nacional de Evaluación de Recursos Naturales.

Skov, F. and Balslev, H. (1989) A revision of Hyospathe (Palmae). Nord. J. Bot. 9, 189-202.

Weberbauer, A. (1945) El mundo vegetal de los Andes peruanos. Lima: Ministerio de Agricultura.

Wessels Boer, J.G. (1968) The geonomoid palms. Verh. Kon. Ned. Akad. Wetensch. Afd. Natuurk., Tweede Reeks 58, 1-202.

Young, K.R. (1990) Biogeography and ecology of a timberline forest in north-central Peru. Boulder: Thesis, Univ. of Colorado.

Young, K.R. (1992) Floristic diversity on the eastern slopes of Peruvian Andes. Candollea 46, $125-43$.

Young, K.R. and León. B. (1988) Vegetación de la zona alta del Parque Nacional Abiseo, Perú. Publ. Museo de Hist. Nat. Ser. B (Botánica) 34, 1-37. 